\title{
Bindestrich-Hermeneutiken - Neue Verortungen der Lektüre?
}

\author{
Hartmut Bleumer · Stephan Habscheid · Constanze Spieß $\cdot$ Niels Werber
}

Angenommen: 10. Dezember 2020 / Online publiziert: 7. Januar 2021

(C) Der/die Autor(en) 2020

Zusammenfassung Brauchen wir eine neue Hermeneutik? - Diese Frage ist rhetorisch und scheint aktuell nur vernünftig zu sein. Doch genau dieser Anschein indiziert erneut jenes Dilemma, in dem sich die aktuelle Lektürepraxis befindet. In dem durch die Digitalisierung beschleunigten Wandel der Lektürepraxis bricht es regelrecht auf, denn in ihm erweist sich die Hermeneutik als ein für Kommunikation und Lektüre unabdingbares ethisches Projekt, das zugleich selbst von eben jener Diversifizierung betroffen ist, die durch die technologisch forcierte Rationalität in Gang gebracht wurde. So zeugt die philosophisch irritierende, germanistische bzw. allgemein literatur- und sprachwissenschaftliche Pluralisierung in vielen fach- oder teilfachspezifischen Hermeneutiken ebenso vom aktuellen Problem der Hermeneutik wie von ihrer Unabdingbarkeit. Die Forderung nach einer Neuen Hermeneutik setzt also zuerst die Diagnose voraus, wie und wo ihre Spielarten sich aktuell verorten, um danach jenen gemeinsamen Ort anzuvisieren, der die Hermeneutik gleichzeitig im > Wir < der Fragestellung zusammenhält. Die dazu möglichen Diagnosen werden wiederum verschieden ausfallen. Doch erst in der Summe ermöglichen sie - so die

\footnotetext{
H. Bleumer $(\square)$

Seminar für Deutsche Philologie, Georg-August-Universität Göttingen, Göttingen, Deutschland E-Mail: hbleume@gwdg.de

S. Habscheid · N. Werber

Germanistisches Seminar, Universität Siegen, Siegen, Deutschland

S. Habscheid

E-Mail: habscheid@germanistik.uni-siegen.de

N. Werber

E-Mail: werber@germanistik.uni-siegen.de

C. Spieß

Institut für Germanistische Sprachwissenschaft, Philipps-Universität Marburg, Marburg, Deutschland E-Mail: constanze.spiess@ uni-marburg.de
} 
These der Herausgeber*innen der Zeitschrift für Literaturwissenschaft und Linguistik - einen Beitrag zur Vergewisserung über das aktuelle Verständnisproblem.

Schlüsselwörter Germanistik · Linguistik · Literaturwissenschaft · Hermeneutik · Neohermeneutik · Diskurshermeneutik · Texthermeneutik · Linguistische Hermeneutik · Literarische Hermeneutik

\section{Hyphen-Hermeneutics: New Localizations of Reading?}

Abstract Do we need a new hermeneutics? - This question is rhetorical and seems only reasonable at the moment. But it is precisely this appearance that once again indicates the dilemma in which current reading practice finds itself. In the transformation of reading practice accelerated by digitalization, it breaks open, for hermeneutics proves to be an ethical project that is indispensable for communication and reading, and at the same time is itself affected by the very diversification that was set in motion by a technologically forced rationality. Thus, the philosophically irritating, Germanistic or general literary and linguistic pluralization into many, subject- or subsubject-specific hermeneutics bears witness to the current problem of hermeneutics as well as to its indispensability. The demand for a New Hermeneutics thus presupposes first of all a diagnosis of how and where its varieties are currently located, in order to then target the common site that simultaneously holds hermeneutics together in the $>$ we $<$ of the question. The possible diagnoses will again be different. But only in their totality - according to the thesis of the editors of the Zeitschrift für Literaturwissenschaft und Linguistik - will they contribute to a reassurance about the current problem of understanding.

Keywords German Studies · Linguistics · Literary Studies · Hermeneutics · Neohermeneutics · Discourse Hermeneutics · Text Hermeneutics · Linguistic Hermeneutics · Literary Hermeneutics

\section{Zum leeren Versprechen der digitalen Vernunft}

Hermeneutik tut not: Angesichts des alltäglichen Scheiterns der Aufklärung in der kulturellen Praxis müsste dies eine ziemlich konsensfähige Formel sein. Man mag ihr schon aufgrund des Eindrucks zustimmen, dass zentrale Kompetenzen im reflektierten Umgang mit Sprache und Text gegenwärtig zu verschwinden scheinen. Vordergründig kann man diesen allgemeinen Wandel des Lektüreverständnisses gewiss für einen der vieldiskutierten Effekte der Digitalisierung halten, der sich insbesondere im Bildungssystem und im Bereich der Populären Kulturen beobachten lässt. Und doch dürfte sich in den oberflächlichen Kompetenzverlusten nur erneut das tiefgreifende Dilemma aufgeklärter Rationalität abzeichnen, in dem sich die alte Macht des Diskurses auf eine geradezu banale Weise bestätigt.

Darum ist es auch riskant, sich diesem Diskurs sofort wieder anzuvertrauen, etwa, indem man den Wandel der sprachlichen und textlichen Möglichkeiten als Verfallsgeschichte erzählt. Immerhin wird man jenseits eines solchen Narrativs und seiner 
impliziten Wertungen sagen dürfen: Wo Sprache und Text nicht mehr durchschaut und hinlänglich beherrscht werden, weil die Begriffe von Lektüre und Textverstehen szientifisch verkürzt werden, wo man sich stattdessen etwa der diskreten Vorauswahl und Lenkung der Algorithmen anvertraut, kommt es zu einer eigentümlichen Sorglosigkeit im Umgang mit Sinnbildungsprozessen. Man könnte sie mit Blick auf das gescheiterte Projekt der Aufklärung fast als Eingang in eine neue, selbstverschuldete Unmündigkeit bezeichnen.

Diese neue Unmündigkeit zeigt sich vielleicht am deutlichsten darin, dass die Heilsversprechen der Algorithmen inzwischen ihre charakteristische Kehrseite offenbaren. Das Versprechen der digitalen Rationalität, die Welt einfach und bequem zugänglich zu machen, führt angesichts der anstrengenden Pluralität, Unverbindlichkeit und Unübersichtlichkeit >postmoderner $<$ Wirklichkeiten zu der verstörten, ja oft genug auch empörten Frage, warum diese so heterogen, kompliziert und schwierig sein müssen. Und wo diese Frage dann auch noch mit weiteren Vereinfachungen beantwortet wird, droht schließlich jener anti-kritische Mythos vollends wirksam zu werden, der sich heute als Benutzerfreundlichkeit tarnt.

Wenn Friedrich Kittler hier aber recht behält, ${ }^{1}$ dann ist er in Wahrheit unfreundlich, weil er die Leser*innen insgeheim über seine Benutzeroberflächen beherrscht und letztlich benutzt. In seinem Zugriff auf die Datenwelt werden die Leser*innen selbst zum Teil des Datensatzes. Die Rationalität des neuen, unangestrengten Lesens gehorcht so einem System, das sie zwar erfunden hat, das ihr dann aber im Gegenzug die Möglichkeit verwehrt, jenseits dieser Rationalität noch weiterzudenken.

Gerade dort, wo dieser Zirkel aufgeht, wird der Lektürebegriff allmählich spürbar defizitär: weil er um die semantische Produktivität seiner Paradoxien und Gegenläufigkeiten verkürzt wird, die es überhaupt erst rechtfertigt, statt vom einfachen Lesen von einer Lektüre zu sprechen. ${ }^{2}$ So wird auch den sorglosesten Leser*innen irgendwann aufgehen, dass ein bloßer Konsum von Texten auf eine sinnlose Rezeptionspraxis herausläuft: da hier Lektüre nur noch simuliert wird. Dieser bedenkliche Hang zur Lektüresimulation ist insbesondere aus der akademischen Lehre gut bekannt. Deutlich werden seine Auswirkungen an der Frustration von Studierenden, die sich lediglich mit Wikipedia-Artikeln auf ihre Stellungnahmen zu literarischen Texten vorbereitet haben, die sie entweder im flüchtigen Lesen nicht verstanden oder aber erst gar nicht gelesen haben - und die dann ihren Lehrveranstaltungen die Schuld dafür geben, Literatur nicht zu verstehen.

Was hier indes nicht verstanden wird, ist das Verstehen selbst. Die Paradoxien des Lesens stürzen diese neuen Leser*innen in Verzweiflung, weil sie inzwischen an scheinbar widerspruchsfreie, systematisch vereinfachende Lösungen gewöhnt sind. Der Prozess der Interpretation erscheint ihrem Lektürehabitus als ein Wagnis zwischen Skylla und Charybdis, nicht nur weil Interpretationen der Literatur in der Dichotomie von richtig und falsch generell nicht aufgehen. Vielmehr spüren die neuen Leser*innen, dass sie im Prozess des Verstehens auch selbst gefragt sind und dabei als Subjekte für ihre Ergebnisse verantwortlich werden. Lektüre lässt sich eben

\footnotetext{
1 Vgl. Kittler, Friedrich: Grammophon. Film. Typewriter. Berlin 1986, S. 3 f.

2 Vgl. Lobsien, Eckhard: Schematisierte Ansichten. Literaturtheorie mit Husserl, Ingarden, Blumenberg. Paderborn/München 2012, S. $11 \mathrm{f}$.
} 
nicht simulieren. Darum geht sie auch in künstlicher Intelligenz nicht auf - wenn nicht schon der Ausdruck der künstlichen Intelligenz eine contradictio in adjecto ist.

$\mathrm{Ob}$ man aus diesen technischen Textproblemen herauskommt, wenn man nur erneut an den kritischen Geist der Aufklärung appelliert oder auch nur an Schleiermachers Minimalbedingung der »Bekanntschaft mit dem Gegenstande und der Sprache $\ll^{3}$ erinnert, ist höchst zweifelhaft. Denn mit guten Gründen kann man argumentieren, dass es letztlich ein übers Ziel hinausschießender, die eigenen rationalen Errungenschaften preisgebender aufklärerischer Rationalitätsimpetus ist, der historisch hinter diesen zirkulären Effekten steckt. So ist der unbeirrte Glaube an eine universale Rationalität offenbar schon für jene alltägliche Empörung mit verantwortlich, aus der heraus einerseits unliebsame Darstellungen der Wirklichkeit als fake news beschimpft, andererseits aber auf der Möglichkeit von >alternativen Fakten< beharrt wird, die jene Unsicherheiten angesichts dieser Wirklichkeit nur wieder vermehren, mit denen sich die Empörung dann erneut befeuern lässt. Intuitiv beutet bereits dieser emotionstheoretisch durchschaubare, hysterische Rückkoppelungsmechanismus eben jenen Orientierungsverlust, der nicht nur technisch-rational erklärlich, sondern auch technisch-rational bedingt ist. Die pure Unvernunft ist eine Spätfolge eines Sinnproblems, das die rationalisierte Vernunft hinterlassen hat. Es zeigt sich bereits in den vermehrt diskutierten Anfängen der philosophischen Hermeneutik im Rahmen der Aufklärung, ${ }^{4}$ wo diese die produktive Offenheit des Sinns mit Hilfe einer mathematisch-notwendigen Vernünftigkeit methodologisch zu eliminieren suchte: in Form eines logisch geschlossenen Auslegungszirkels, dessen Mangel die philosophische Hermeneutik schon einmal neu begründet hat. ${ }^{5}$

\section{Von der Eigenvernunft interpretierender Kommunikation}

Wenn es heute als zeitgemäß und (dem*der naiven Betrachter*in) als möglich erscheint, hermeneutische Traditionen etwa zugunsten der sprachstatistischen Analyse großer Textkorpora oder der maschinellen Sprachverarbeitung ganz zu überwinden, mag dies freilich auch der nicht selten zu beobachtenden »puren Unvernunft«

\footnotetext{
3 Schleiermacher, Friedrich Daniel Ernst: Hermeneutik und Kritik. Mit einem Anhang sprachphilosophischer Texte Schleiermachers. Hg. und eingeleitet von Manfred Frank. 7. Aufl. Frankfurt a. M. 1999, S. 75 .

4 Vgl. Bühler, Axel (Hg.): Unzeitgemäße Hermeneutik. Verstehen und Interpretation im Denken der Aufklärung. Frankfurt a. M. 1994; Bühler, Axel/Cataldi Madonna, Luigi (Hg.): Hermeneutik der Aufklärung (=JSTOR 8, 2 1994); Beetz, Manfred/Cacciatore, Guiseppe (Hg.): Die Hermeneutik im Zeitalter der Aufklärung. Köln u. a. 2000; Danz, Christian: Schelling und die Hermeneutik der Aufklärung. Tübingen 2012.

5 Vgl. daher das Stichwort der >neuen Hermeneutik macher bei Weimar, Klaus: Art. >Hermeneutik1<. In: Reallexikon der deutschen Literaturwissenschaft. Neubearbeitung des Reallexikons der deutschen Literaturgeschichte. Hg. von Harald Fricke. Berlin/New York 2007, S. 25-29; Bogdal, Klaus-Michael: »Problematisierung der Hermeneutik im Zeichen des Poststrukturalismus «. In: Heinz Ludwig Arnold/Heinrich Detering (Hg.): Grundzüge der Literaturwissenschaft. München 1996, S. 137-156; Jung, Werner: »Neuere Hermeneutikkonzepte. Methodische Verfahren oder geniale Anschauung?« In: Klaus Michael Bogdal (Hg.): Neue Literaturtheorien. Eine Einführung. Opladen 1997 [1990], S. 159-180.
} 
(s. oben) im Umgang mit Texten geschuldet sein, der in immer noch wirkmächtigen postmodernen Interpretationstheorien als Freiheit der Leser*innen gerechtfertigt, gefeiert und gefordert wurde. ${ }^{6}$ Dabei drohte in Vergessenheit zu geraten, dass eine wohl verstandene Hermeneutik im griechisch-lateinischen Rationalismus wurzelt und - im Gegensatz zur bis heute (etwa in Verschwörungstheorien) lebendigen vorantiken »Hermetik« - den strengen wissenschaftstheoretischen Anspruch der Falsifizierbarkeit einlöst, und zwar auf der Grundlage eines »Sozialvertrags«, der einen vernünftigen Umgang mit der Textintention (intentio operis) sicherstellt und begrenzt. ${ }^{7}$

So weist Umberto Eco in einer Klärung des Begriffs der Textintention - in Abgrenzung sowohl von der Intention des empirischen Autors (intentio auctoris) als auch zur jeweiligen Intention, die ein empirischer Leser versteht (intentio lectoris) im Rahmen einer (durchaus normativen) semiotischen Hermeneutik darauf hin, dass »die Wörter des Autors ein ziemlich sperriges Bündel handfester Indizien [bilden], die der Leser nicht einfach schweigend oder palavernd übergehen darf $^{8}$. Daraus kann gefolgert werden: »Zwischen der unergründlichen Intention des Autors und der anfechtbaren Intention des Lesers liegt die transparente Textintention, an der unhaltbare Interpretationen scheitern. $\ll^{9}$ Eine so verstandene hermeneutische Textinterpretation, die nicht zuletzt auf der systematischen Grundlage einer linguistischen Theorie der Textkommunikation ${ }^{10}$ entfaltet werden kann, muss also gar nicht empirisch und logisch gebändigt werden, da sie dies - vernünftig betrieben - in gewisser Weise per se bereits ist. $^{11}$

Vor diesem Hintergrund mag es verständlich erscheinen, wenn diejenigen, die sich heute noch (oder wieder) affirmativ auf >hermeneutische< Grundlagen beziehen, durch eine spezifische >Bindestrich-Bezeichnung<verdeutlichend markieren, dass nicht etwa irgendeine landläufige Art von Hermeneutik gemeint ist, sondern eine bestimmte wissenschaftliche Spielart, deren Charakteristik das Attribut aussagt,

\footnotetext{
6 Vgl. etwa: »Ein Text ist wie ein Picknick, zu dem der Autor die Wörter und der Leser den Sinn beisteuert« (T. Todorow, zitiert nach Eco, Umberto: Zwischen Autor und Text. München 1996 [1992], S. 29f.).

7 Ebd., S. 33, 87.

8 Ebd., S. 30.

9 Ebd., S. 87.

10 Vgl. dazu Hausendorf, Heiko u. a.: Textkommunikation. Ein textlinguistischer Neuansatz zur Theorie und Empirie der Kommunikation mit und durch Schrift. Berlin/Boston 2017.

11 Wer für den Anspruch der Wissenschaftlichkeit Weiteres fordert (etwa Formalisierung, strenge Deduktion, experimentelle Wiederholbarkeit, objektive Methodik etc.), steht vor der in den Sprach- und Literaturwissenschaften kaum einzulösenden Herausforderung zu zeigen, dass diese Merkmale für die eigene wissenschaftliche Praxis nicht nur partiell, sondern aufs Ganze gesehen gelten. Vgl. Hermanns, Fritz: »Linguistische Hermeneutik. Überlegungen zur überfälligen Einrichtung eines in der Linguistik bislang fehlenden Teilfaches«. In: Ders.: Der Sitz der Sprache im Leben: Beiträge zu einer kulturanalytischen Linguistik. Hg. von Heidrun Kämper/Angelika Linke/Martin Wengeler. Berlin/Boston 2012, S. 67-102, hier S. 98 [Erstmals erschienen in: Angelika Linke/Hanspeter Ortner/Paul R. Portmann-Tselikas (Hg.): Sprache und mehr. Ansichten einer Linguistik der sprachlichen Praxis. Tübingen 2003, S. 125-163.]; Ders.: »Diskurshermeneutik«. In: Ders.: Der Sitz der Sprache im Leben: Beiträge zu einer kulturanalytischen Linguistik. Hg. von Heidrun Kämper/Angelika Linke/Martin Wengeler. Berlin/Boston 2012, S. 104-126, hier S. 110 [Erstmals erschienen in: Ingo Warnke (Hg.): Diskurslinguistik nach Foucault. Theorie und Gegenstände. Berlin/New York 2007, S. 187-210.].
} 
man denke etwa an die semiotische Hermeneutik, linguistische Hermeneutik, objektive Hermeneutik, Tiefenhermeneutik, sozialwissenschaftliche Hermeneutik, Alltagshermeneutik und Diskurshermeneutik, um nur einige zu nennen. Auch die als Neo-Hermeneutik gekennzeichneten Ansätze in der Literaturwissenschaft (s. unten) stellen Tradition und Innovation gleichermaßen heraus, auch wenn die Bezeichnung noch keinen Hinweis darauf gibt, an welche Wissensbestände angeknüpft wird und in welche Richtung die Reise gehen soll.

Jedenfalls scheinen aber einer szientifisch begründeten Ablehnung der Hermeneutik in Teilen der Geistes- und Sozialwissenschaften heute vielfältige Ansätze u. a. in den Sprach- und Literaturwissenschaften gegenüber zu stehen, die Hermeneutik als wissenschaftliche Praxis mit hoher einzelfachlicher, interdisziplinärer und gesellschaftlicher Aktualität und Relevanz ${ }^{12}$ theoretisch und empirisch, zumal in Anbetracht der sich verändernden Kommunikations- und Medienverhältnisse und der hiermit verbundenen methodischen und forschungspraktischen Potentiale für die Wissenschaft selbst, weiterzuentwickeln versuchen (etwa unter dem Stichwort mixed methods).

\section{Wissenschaftliche Verdrängungsprozesse und ihre Selbstbeschleunigung}

Die Vorstellung, dass die Hermeneutik durch sciences ersetzt werden könne und müsse, dürfte nicht selten auch auf einer fundamentalen Unklarheit darüber beruhen, was überhaupt genau unter >Hermeneutik $<$ zu verstehen ist. Wie Fritz Hermanns auf der Basis semantischer Analysen zeigt, bezieht sich der Ausdruck Hermeneutik auf ein

»Konglomerat heterogenster Entitäten, nämlich von Ereignissen (Verstehensereignissen), Tätigkeiten (Erklären und Lehren), Fähigkeiten (Künsten des Verstehens und Erklärens), Handlungsanweisungen (Regeln) und Wissensbeständen (Theorien, Theoriefragmenten), sofern sie nur etwas mit Verstehen zu tun haben. Dieser Bezug macht die einzelnen Bedeutungen des Wortes Hermeneutik metonymisch (zueinander). $\ll^{13}$

Es kommt dabei weniger auf die Heterogenität der Entitäten als auf den Zusammenhang an, der hermeneutische Praxis und ihr Resultat, Methodenlehre, Empirie und Theorie (potentiell) miteinander verbindet: Verstehen seinerseits fasst Hermanns als ein zweistelliges Prädikat, dass bestimmte Relationen des Erkennens von etwas als etwas anderem aussagt, und zwar weit über kommunikative Erscheinungsformen hinaus, wie sie etwa im Mittelpunkt sozialwissenschaftlicher Auffassungen von Hermeneutik stehen: ${ }^{14}$ »Ein Erkennen nennen wir vor allem dann Verstehen, wenn

\footnotetext{
12 Allgemein hierzu Hermanns: »Linguistische Hermeneutik« (s. Anm. 11), S. 67.

13 Ebd., S. 73.

14 Vgl. dazu Knoblauch, Hubert: Wissenssoziologie. Konstanz 2005, S. 176-182.
} 
Zusammenhänge, Gründe und Ursachen, Zwecke und Funktionen, Bedeutungen und Sinn erkannt werden. $\ll^{15}$

Während ein schlichtes Wiedererkennen den unproblematischen Fall darstellt, wird es stets dann anspruchsvoll, wenn sowohl das zu Erkennende als auch die zum Verstehen (neben anderem) erforderlichen Wissensbestände erst (um-)konstruiert werden müssen. Vom Verstehen (als Ereignis und Ergebnis) zu unterscheiden ist nach Hermanns das Interpretieren, eine kommunikative, wahrnehmbare Handlung, die einem anderen das (eigene) Verstehen zu einem bestimmten Zweck erklären soll, sowie das Anwenden von Texten bei der kontextspezifischen Herstellung von Entscheidungen, etwa im Recht oder in der die Schrift auslegenden Predigt. ${ }^{16}$

Vor diesem Hintergrund einer derartigen Begriffsklärung muss die Wilhelm Dilthey zugeschriebene, begrifflich schiefe Opposition zwischen Verstehen als geisteswissenschaftlichem und Erklären als naturwissenschaftlichem modus operandi als abwegig erscheinen, wie Hermanns schlüssig ausführt:

»Die beiden Begriffe können überhaupt kein Gegensatzpaar bilden, weil es sich bei ihnen gar nicht um Kohyponyme handelt. Auch in allen Naturwissenschaften geht es selbstverständlich darum, etwas zu verstehen (u. a. Kausalzusammenhänge), und die Kulturwissenschaften können umgekehrt natürlich nicht darauf verzichten, die von ihnen dargestellten Phänomene zu erklären, d. h. verständlich zu machen. «17

All dies ist durch und durch rational. Die Folgen, die dagegen ein in seinem überbordenden Rationalitätsglauben naiv anmutender Bändigungsversuch des Sinns für das Verstehen hat, sind heute alltäglich an der unversöhnlichen Agonie jener Lektüren ablesbar, in denen Verstehen und Verständnis erst gar nicht mehr gesucht werden. Darum ist es auch nicht einzusehen, warum, nachdem auch die aufklärerische Verheißung der neuen digitalen Freiheiten des Netzes gescheitert ist, diese ausgerechnet durch die Aufklärung über deren Risiken gebannt werden soll. Beobachtbar wird dieses Risiko immerhin mit Hilfe der klassischen Systemtheorie. Aber auch sie stößt an ihre epistemische Grenze. Ihr fehlt durch ihr notorisches Spannungsverhältnis zur Hermeneutik, in dem sie deren Paradoxien erneut eher simuliert als nachvollzieht, die letzte Möglichkeit, das Sinnproblem zu interpretieren. ${ }^{18}$ Gleichwohl wird gerade auch in ihren neueren, literaturwissenschaftlichen Varianten wieder deutlich: Dem aufklärenden Zirkel fehlt etwas, darum reproduziert er in seiner Beschleunigung nur sein Defizit. Das immer höhere Tempo der Informationsbewegungen ist nicht nur eine Reaktion auf den Sinnverlust, es treibt dessen Inflation selbst mit voran. Poin-

\footnotetext{
15 Hermanns: »Linguistische Hermeneutik« (s. Anm. 11), S. 79.

16 Vgl. ebd., S. $72 \mathrm{f}$., $88 \mathrm{f}$.

17 Ebd., S. 89.

18 Vgl. die Kritiken am systemtheoretischen Grundmodell in de Berg, Henk/Prangel, Matthias (Hg.): Systemtheorie und Hermeneutik. Tübingen 1997. Für eine Annäherung plädiert in der Soziologie Schneider, Wolfgang Ludwig: »Hermeneutik und Systemtheorie«. In: Ders.: Grundlagen der soziologischen Theorie. Bd. 3: Sinnverstehen und Intersubjektivität - Hermeneutik, funktionale Analyse, Konversationsanalyse und Systemtheorie. 2. Aufl. Wiesbaden 2009, S. 143-292. Zu den Weiterentwicklungen im literaturwissenschaftlich-systemtheoretischen Grundverständnis vgl. dagegen Werber, Niels (Hg.): Systemtheoretische Literaturwissenschaft. Begriffe - Methoden - Anwendungen. Berlin u. a. 2011.
} 
tiert gesagt: Letztlich ist schon eine völlig aufgeklärte Rationalität in ihrer strikten Funktionalität sinnlos, ebenso wie schließlich ein bloß technisch-rationales System den Sinn nur simulieren kann, den es beobachten möchte. Mit dem kritischen Denken, dem die Aufklärung vollends zum Durchbruch verholfen hat, lassen sich so gewiss immer wieder nicht nur die Grenzen der Aufklärung, sondern auch die ihrer Rationalität bewusst halten. Aber es kommt - im öffentlichen Diskurs wie im Umgang mit Literatur - offenbar auf gerade jenen Sinn an, den diese Rationalität über ihre eigene epistemische Grenze verschoben hat, der also - namentlich mit der Transzendentalphilosophie Kants - durch diese Rationalität vollends transzendent geworden ist.

\section{Lektürepraxis und Neue Hermeneutik: Symptome einer Krise?}

Nun scheint ein solcher argumentativer Sprung von den alten Höhen des akademischen Elfenbeinturms auf den unsicheren Boden der neuen Tatsachen gewiss als gewagt: Dass Lehrer*innen und Pädagog*innen in der alltäglichen schulischen Praxis beobachten, wie Schüler*innen sich um die Ausbildung ihrer individuellen Lektürekompetenz betrügen lassen, weil bereits die von den Promotoren der Digitalisierung für den Unterricht produzierten Lehrmaterialien unterstellen, dass ihre Memorial- und Imaginationsmöglichkeiten vom Smartphone beherrscht werden und so jedes mögliche Niveau von vorneherein unterlaufen; dass in der akademischen Lehre die allgemeine Erosion jenes Schreib-, Ausdrucks- und Interpretationsvermögens beklagt wird, das den jungen, hoffnungsfrohen Intelligenzen das eigene Verständnis für die Traditionen der bisherigen wissenschaftlichen Praxis erst eröffnen kann; dass die Vernetzungen der digitalen Medien ihren Nutzer*innen eine Informationsfreiheit versprechen, die sie ihnen mit ihren konditionierenden Selektions-, Bild- und Textmechanismen sogleich in Notwendigkeit verwandeln; dass durch diese Selektions-, Bild- und Textmechanismen im Bereich der populären Kulturen schließlich angesichts der vollständig agonalen Wirklichkeitssimulationen zu politischen Fragen sogar der Eindruck entsteht, das Verstehen einer gemeinsamen Wahrheit sei unmöglich, als ginge es nur darum, die jeweils beste mögliche Wirklichkeitssicht durchzusetzen - all dies ergibt eine Gemengelage, die von den intellektuellen Weichenstellungen doch sehr weit absteht, die sich mit dem Rationalitätsproblem der Aufklärung und der Relevanz der Hermeneutik für den Text- und Lektürebegriff verbinden.

Aber auch angesichts dieses Abstandes dürfte gelten: Das Einfachheitsversprechen einer kurzschlüssig-geschlossenen Erklärung wäre eine rationale Täuschung darum scheint ein Einspruch der alten Hermeneutik in diese neue Gemengelage legitim. Freilich ist an diesem Einwand gegen den historischen Abstand von Begriff und Kulturphänomen mindestens dies richtig: Die alte Hermeneutik wird sich angesichts der neuen Lektürepraxis und ihren agonalen Folgen ebenso neu wenden lassen müssen.

Die Forderung nach einer solchen Hermeneutik angesichts des neuen Lesens ist allerdings zumindest in Teilen ziemlich wohlfeil. Denn nicht nur das Etikett der Neuen Hermeneutik gibt es längst, auch hat sich die damit angedeutete Wende bereits 
vollzogen. Nur geht diese Wende der Hermeneutik irritierenderweise weder in dem neuen Etikett auf, noch ist sie entschieden auf die Praxis des neuen Lesens bezogen. Der Terminus der >Neo-Hermeneutik < findet sich entweder in einer auf die genuin literaturwissenschaftliche Autorschaftsdiskussion verengten Extension oder aber in der theologisch-existenzialistischen Variante als Postulat einer New Hermeneutic. ${ }^{19}$

Dies mag es gerechtfertigt erscheinen lassen, dem postmodernen Impuls nachzugeben und den Universalitätsanspruch der Hermeneutik aufzugeben, um stattdessen nur noch von Hermeneutiken im Plural zu sprechen. Allerdings ist damit das Methodenverständnis der philosophischen Hermeneutik zugunsten eines wissenschaftlichen Methodologieverständnisses aufgelöst, das es eher sinnvoll erscheinen lässt, statt von Hermeneutik von einer Metatheorie zu verschiedenen Interpretationstheorien zu sprechen. ${ }^{20}$ Mit dem reinen Theorieanspruch manövriert man sich so in eine Sackgasse. Darum wird man zuerst, am naheliegendsten vorrangig für die alltägliche akademische Lehrpraxis, nach dem praktischen Einfluss der traditionellen, nachaufklärerischen Hermeneutik heute und dem aktuellen Grad des hermeneutischen Bewusstseins fragen müssen. Dass man auch diese bereits als Neue Hermeneutik bezeichnet hat, war schon festzuhalten. Doch trotz der Etikettierung des vermeintlich $>$ Neuen< lässt sich im universitären Alltagsgeschäft weiterhin beobachten, dass schon das Stichwort der Hermeneutik jedenfalls unter Germanisten immer noch kritische Ressentiments auslöst.

\subsection{Linguistische Aspekte: Zur Unhintergehbarkeit der Hermeneutik(en)}

In der Germanistischen Linguistik stehen jenen Diskursgemeinschaften, denen eine hermeneutische Reflexion ihres Tuns (mehr oder weniger tief gehend) vertraut ist, Forschungsszenen gegenüber, die sich angesichts der Hermeneutik völlig indifferent, wenn nicht ablehnend positionieren. Gründe hierfür liegen - neben den bereits

\footnotetext{
19 Vgl. den Terminus der Neo-Hermeneutik mit Blick auf das Intentionalismusproblem bei Köppe, Tilmann/Winko, Simone: Neuere Literaturtheorien. Eine Einführung. 2., aktualisierte und erweiterte Auflage. Stuttgart/Weimar 2013, S. 133-148. Siehe unten, Anm. 46. Im Bereich der Theologie vgl. Thiselton, Anthony C.: »The New Hermeneutic«. In: I. Howard Marshall (Ed.): New Testament Interpretation. Essays on Principles and Methods. Carlisle 1992 [1977], S. 308-333; Ders.: New Horizons in Hermeneutics. The Theory and Practice of Transforming Biblical Reading. Grand Rapids, Mich. 1998; Ders.: Hermeneutics. An Introduction. Grand Rapids, Mich. u. a. 2009. Vgl. auch Wilkens, Lorenz: Hermeneutik nach dem Existentialismus. Theologische Vorträge und Aufsätze. Frankfurt a. M. 2007. Vgl. für das erstgenannte Etikett in der Germanistik auch in den literaturwissenschaftlichen Überblicken bei Berensmeyer, Ingo: »Methoden hermeneutischer und neohermeneutischer Ansätze«. In: Vera Nünning/Ansgar Nünning (Hg.): Methoden der literatur- und kulturwissenschaftlichen Textanalyse. Ansätze - Grundlagen - Modellanalyse. Stuttgart/Weimar 2010, S. 29-50, hier S. 47 f., der interessanterweise dessen weitgehende Wirkungslosigkeit konstatiert. Darum scheint es durchaus erfolgversprechend, die hermeneutische Methode unabhängig vom Intentionalismusproblem in der Autorschaftsdebatte, nämlich mit dem weiteren Blick auf die Philosophie Manfred Franks (siehe unten, Anm. 57), zu verwenden, vgl. Gnüg, Hiltrud: »Hermeneutik/Neohermeneutik«. In: Jost Schneider (Hg.): Methodengeschichte der Germanistik. Berlin 2009, S. 225-254, hier S. 152. Vgl. zuvor Leiteritz, Christiane: »Hermeneutische Theorien«. In: Martin Sexl (Hg.): Einführung in die Literaturtheorie. Wien 2004, S. 129-159, hier S. 152.

${ }^{20}$ Vgl. Danneberg, Lutz: Hermeneutiken. Bedeutung und Methodologie. Mit einem Vorwort von Andrea Albrecht und Carlos Spoerhase. Berlin/Boston u. a. 2019. Das Problem auch in: Jannidis, Fotis u. a. (Hg.): Hermeneutics and philosophy of science. Berlin/Boston 2016 (Sonderheft des Journal of literary theory 10,1 [2016]).
} 
skizzierten allgemeinen szientifischen Missverständnissen - fachspezifisch in der gewohnten Gegenüberstellung einer Langue- bzw. Kompetenz-Linguistik, die sich für (universelle) »Gesetze, Formen und Strukturen $\aleph^{21}$ des Sprachsystems interessiert, und einer >Parole-Linguistik<, die den Gebrauch sprachlicher Zeichen in der Kommunikation in den Mittelpunkt stellt. ${ }^{22}$ Da Zeichen unter den Gesichtspunkten ihrer Materialität und Operativität in der Kommunikation als >Medien $<$ zu betrachten sind ${ }^{23}$ fächert sich der abstrakte Gegenstand >Sprache< dabei nach diversen medialen Existenzweisen auf, wovon die an den menschlichen Körper gebundene Interaktion und sprachliche Ko-Operation ${ }^{24}$ unter räumlich und zeitlich Anwesenden einen speziellen Fall unter anderen darstellt. Diesem können unter anderem die auf Schrift und >Lesbarkeit< beruhenden Existenzweisen von Sprache an die Seite gestellt werden, ${ }^{25}$ die ihrerseits unter dem Gesichtspunkt ihrer Situationalität medial weiter aufzufächern sind.

Während es im Bereich der Parole selbst um Prozesse der Verständigung geht, die es sprachtheoretisch zu erklären gilt, ${ }^{26}$ was eine zumindest auch hermeneutische Vorgehensweise nahelegt, scheint die Langue-Linguistik - etwa im Bereich der apparativen Messung von Lauten oder der auf reine Oberflächenphänomene bezogenen statistischen Auswertung von Korpusdaten ${ }^{27}$ - auf den ersten Blick ohne Hermeneutik auskommen zu können. Zu bedenken ist allerdings, dass bereits das sprachliche Zeichen selbst ein zweistelliges ist, so dass Ausdruck und Inhalt in der Regel nicht unabhängig voneinander betrachtet werden können. ${ }^{28}$ Zudem stellt, wie oben bereits ausgeführt, jede Begründung eines Gegenstands einen hermeneutischen Akt dar. Werden etwa in der Grammatik (kommunikative) Funktionen bzw. in der Lexikologie Bedeutungen thematisiert bzw. erklärt, ist der interpretierende Anteil zwar oft nur implizit, aber doch unverzichtbar. ${ }^{29}$

Die Linguistik benötigt die Hermeneutik also sowohl auf Grund ihres Gegenstands als auch in methodologischer und theoretischer Hinsicht. ${ }^{30}$ Dementsprechend gibt es spätestens seit den 1970er-Jahren eine hermeneutische Reflexion, etwa bei

\footnotetext{
21 Busse, Dietrich/Teubert, Wolfgang (1994): »Ist Diskurs ein sprachwissenschaftliches Objekt? Zur Methodenfrage der historischen Semantik«. In: Dies./Fritz Hermanns (Hg.): Begriffsgeschichte und Diskursgeschichte. Methodenfragen und Forschungsergebnisse der historischen Semantik. Opladen 1994, S. 10-28, hier S. 12, zitiert nach Bär, Jochen A.: Hermeneutische Linguistik. Theorie und Praxis grammatisch-semantischer Interpretation. Grundzüge einer Systematik des Verstehens. Berlin/München/Boston 2015, S. 2.

22 Vgl. Bär (s. Anm. 21), S. 2.

23 Vgl. Schneider, Jan Georg: »Medien als Verfahren der Zeichenprozessierung: Grundsätzliche Überlegungen zum Medienbegriff und ihre Relevanz für die Gesprächsforschung«. In: Gesprächsforschung Online-Zeitschrift zur verbalen Interaktion 18 (2017), S. 34-55.

24 Anthropologisch grundlegend Goodwin, Charles: Co-Operative Action. New York 2018.

25 Vgl. Hausendorf u. a. (s. Anm. 10).

26 Vgl. exemplarisch Knobloch, Clemens: Sprache und Sprechtätigkeit. Sprachpsychologische Konzepte. Tübingen 1994.

27 Vgl. Hermanns: »Linguistische Hermeneutik« (s. Anm. 11), S. 96.

28 Vgl. Bär (s. Anm. 21), S. 10.

29 Vgl. Hermanns: »Linguistische Hermeneutik« (s. Anm. 11), S. 96.

30 Vgl. ebd., S. 70. Zur Forschungsgeschichte im Folgenden vgl. ebd., S. 69.
} 
Ludwig Jäger im Rahmen einer kulturwissenschaftlichen Linguistik, die an die sprachtheoretische Tradition der Aufklärung und Romantik anknüpft, ${ }^{31}$ oder bei Rudi Keller im Kontext der Sprechakttheorie, ${ }^{32}$ die sich mit dem Verstehen und der Interpretation sprachlichen Handelns befasst. Seit den 1980er-Jahren werden hermeneutische Traditionen in der Linguistik weiter aufgearbeitet und fachspezifisch ergänzt. ${ }^{33} \mathrm{Zu}$ ihren Gegenständen gehören neben den Kernbereichen des Sprachsystems etwa Texte und Textsorten, Gespräche und Gesprächssorten, sprachliche Praktiken, Begriffe und Diskurse, jeweils abstrakt wie auch im Blick auf den bemerkenswerten Einzelfall (z. B. eine politische Rede), ${ }^{34}$ auch wenn man sich bei Einzeltextinterpretationen (wie in vielen anderen Bereichen der wissenschaftlichen Arbeit auch) mit dem Wahrheitsanspruch intersubjektiver, argumentativ hergestellter Plausibilität begnügen muss. ${ }^{35}$

Der in solchen Kontexten erhobenen programmatischen Forderung, eine »Linguistische Hermeneutik « als interdisziplinär vernetztes Teilfach der Linguistik zu etablieren, steht heute (wieder) der Versuch gegenüber, die gesamte Sprachwissenschaft als »Hermeneutische Linguistik « von diesem Standpunkt aus zu begründen. ${ }^{36}$ So arbeitet Jochen Bär heraus, dass selbst ein genuin linguistischer Gegenstandsbereich wie die strukturalistisch fundierte historische Semantik - und zwar gerade auch dort, wo sie sich streng an durch grammatische Konstruktionen systematisch strukturierte Kollokationen der lexikalischen Zeichen hält - weder objektive Strukturen noch das Wissen der am historischen Kommunikationsprozess Beteiligten erfassen kann, sondern dass sie stets das historische sprachliche System von der Warte des linguistischen Interpreten in der Gegenwart aus beschreibt und erklärt:

»Ein Zeichen hat aus Sicht der historischen Semantik nicht per se Bedeutung, sondern erhält sie immer nur durch die Deutung einer rezipierenden Instanz. Die Bedeutung eines sprachlichen Zeichens, wie sie die historische Semantik angeben kann, ist der von ihr auf der Grundlage philologischen Sprachwissens strukturierte Kotext des Zeichens: das Ensemble anderer Zeichen, mit denen das Zeichen nach ihrer Deutung funktional verknüpft ist, will sagen: auf die es nach bestimmten von ihr gesehenen Regeln verweist. $\ll^{37}$

Ebenfalls seit den 1970er-Jahren setzt sich die Germanistische Linguistik mit genuin sozialwissenschaftlichen Ansätzen auseinander, die den Begriff der Interak-

\footnotetext{
31 Vgl. z. B. Jäger, Ludwig: »Zu einer hermeneutischen Begründung der Sprachtheorie. Historisch-systematische Skizze«. In: Germanistische Linguistik 5/6 (1977), S. 3-78.

32 Vgl. Keller, Rudi: »Verstehen wir, was ein Sprecher meint, oder was ein Ausdruck bedeutet? Zu einer Hermeneutik des Handelns«. In: Klaus Baumgärtner (Hg.): Sprachliches Handeln. Heidelberg 1997, S. $1-27$.

${ }^{33}$ Vgl. u. a. Biere, Bernd Ulrich: Verständlich-Machen. Hermeneutische Tradition - historische Praxis sprachtheoretische Begründung. Tübingen 1989; Hermanns, Fritz/Holly, Werner (Hg.): Linguistische Hermeneutik. Theorie und Praxis des Verstehens und Interpretierens. Tübingen 2007; Hermanns: »Linguistische Hermeneutik « (s. Anm. 11).

34 Vgl. ebd., S. 97; Hermanns: »Diskurshermeneutik« (s. Anm. 11), S. 114.

35 Vgl. Hermanns: »Linguistische Hermeneutik« (s. Anm. 11), S. 98.

36 Vgl. Bär (s. Anm. 21).

37 Ebd., S. 37.
} 
tion bzw. der Kommunikation ins Zentrum rücken und diese Phänomene von dem in ihnen situierten Zeichengebrauch her als >sinnhaftes soziales Geschehen ${ }^{38}$ verstehend zu ergründen versuchen. ${ }^{39}$ Obwohl es sich bei solchen Gegenständen im Kern um Prozesse der Verständigung handelt, verfügen nicht alle diese Ansätze über eine angemessene Theorie der Interpretation. ${ }^{40}$ Dies gilt etwa für die Konversationsanalyse, die im Zusammenhang mit Interpretationsproblemen einer kontrollierten Erweiterung um eine ethnographische Komponente bedarf. ${ }^{41}$ Umgekehrt setzen sich die deutschsprachigen Sozialwissenschaften seit den 1970er-Jahren mit Problemen der Hermeneutik auseinander, teils im Blick auf strukturelle, >objektive< gesellschaftliche Wissensbestände, die als unbewusste, latente (Tiefen-)Sinnstrukturen in der Interaktion reproduziert werden (>Objektive Hermeneutik $<$ ), teils hinsichtlich einer methodologisch reflektierten Rekonstruktion des (alltäglichen) Sinns, den die Beteiligten im Rekurs auf mitgebrachtes Wissen immer erst gemeinsam miteinander hervorbringen ( >sozialwissenschaftliche Hermeneutik<, > hermeneutische Wissenssoziologie $<$ ). ${ }^{42}$

In der medialen Gegenwart stellen mehr oder weniger menschenähnliche, sprachverarbeitende Technologien eine besondere theoretische Herausforderung für die linguistische und bzw. als sozialwissenschaftliche Hermeneutik dar, deren virtuelle >Dialogfähigkeit< und >Handlungsträgerschaft< zur Diskussion stehen: ${ }^{43}$ Für deren Erklärung bedarf es einer Beschreibungssprache, die anthropozentrische Festlegungen ebenso vermeidet wie die voreilige Symmetrisierung menschlicher und technischer Beiträge und die in der Tradition der Workplace Studies die Verwobenheit von Technik mit menschlicher (Hintergrund-)Kooperation konsequent empirisch zum Thema macht. ${ }^{44}$

\footnotetext{
38 Hausendorf u. a. (s. Anm. 10), S. 229.

39 Vgl. Knoblauch (s. Anm. 14), S. 176.

40 Vgl. Deppermann, Arnulf: »Ethnographische Gesprächsanalyse: Zu Nutzen und Notwendigkeit von Ethnographie für die Konversationsanalyse«. In: Gesprächsforschung - Online-Zeitschrift zur verbalen Interaktion 1 (2000), S. 96-124, hier S. 96, mit Blick auf die Konversationsanalyse.

41 So Deppermann, ebd., und ders.: »Analytikerwissen, Teilnehmerwissen und soziale Wirklichkeit in der ethnographischen Gesprächsanalyse«. In: Ders./Martin Hartung (Hg.): Gesprochenes und Geschriebenes im Wandel der Zeit. Festschrift für Johannes Schwitalla. Mannheim 2015, S. 32-59. Im Kontext der germanistischen Diskussion um eine Linguistische Hermeneutik bzw. Hermeneutische Linguistik spielt die Ethnographie entgegen ihrer Relevanz in der internationalen Tradition der linguistischen Anthropologie insgesamt nur eine marginale Rolle.

42 Vgl. Knoblauch (s. Anm. 14), S. 176-182.

43 Vgl. sozialtheoretisch grundlegend Krummheuer, Antonia: Interaktion mit virtuellen Agenten? Zur Aneignung eines ungewohnten Artefakts. Stuttgart 2010.

44 Vgl. im Grenzbereich zwischen Sozio-informatik und Angewandter Linguistik Habscheid, Stephan u. a.: »Professionelle Emotionalität und humanoide Robotik in der institutionellen Kommunikation«. In: Rudolf de Cillia/Helmut Gruber/Jürgen Spitzmüller (Hg.): Institutionelle und organisationale Kommunikation. Theorie, Methodologie, Empirie und Kritik. Gedenkschrift für Florian Menz. Göttingen 2020, S. 169-188.
} 


\subsection{Literaturwissenschaftliche Aspekte: Zum methodischen Defizit der Theorie}

In der Literaturwissenschaft gelten die Ressentiments gegenüber der Hermeneutik oft dem vermeintlichen Traditionalismus der alten Hermeneutik. Diese Ressentiments sind dabei jedoch ihrerseits ziemlich traditionell, d. h. aus der eigenen Fachgeschichte heraus verständlich, in die zunächst der Historismus als die für die deutsche Geisteswissenschaft typische Sonderform des Geschichtsdenkens hineinspielt, in der dann, über die historische Hermeneutik Johann Gustav Droysens, der Begriff der >Wirkungsgeschichte $<$ bei Hans-Georg Gadamer wichtig wird. Dabei erscheint vor allem die durch den Einfluss Martin Heideggers pointierte, ontologische Grundlage des Verstehens in einem kritischen Licht, so als ob dadurch auch der von Gadamer proklamierten >Horizontverschmelzung « zwischen Interpret und historischem Gegenstand ein unverrückbares semantisches Moment zugeschrieben würde. Auch ist nicht immer klar, ob dort, wo die philosophische Hermeneutik Gadamers immer noch pauschal kritisch angesprochen wird, damit nicht eigentlich nur die alte Interpretationspraxis im Sinne Emil Staigers vor Augen steht. ${ }^{45}$

Dass bei alldem auch noch der Vorwurf eines verkappten naiven Intentionalismus droht, durch den der Leser letztlich doch nur wieder darauf verpflichtet würde, das zu verstehen, was der Autor einmal historisch hätte sagen wollen, bleibt zwar unausgesprochen. Ohne dieses Vorurteil gegen die geisteswissenschaftliche Hermeneutik ist es aber wohl nicht zu verstehen, warum die Hermeneutik letztlich in der Autorschaftsdiskussion - unter dem Stichwort der Neo-Hermeneutik - so prominent wieder aufgetaucht ist. ${ }^{46}$

Wenigstens anmerken muss man dazu immerhin, dass der Traditionalismusvorwurf außerhalb des europäisch-geisteswissenschaftlichen Denkrahmens offenbar wenig Evidenz besitzt. Dies hat vermutlich damit zu tun, dass die angelsächsische Geschichtsphilosophie die Traditionsorientierung des Historismus angesichts des Begriffs der Wirkungsgeschichte nicht automatisch mitdenkt und ihn daher auch deutlich leichter genau gegenläufig zu wenden vermag. Sonst hätte die Hermeneu-

\footnotetext{
45 Vgl. Droysen, Johann Gustav: Historik. Historisch-kritische Ausgabe von Peter Leyh. Bd. 1: Rekonstruktion der ersten vollständigen Fassung der Vorlesungen (1857). Grundriß der Historik in der ersten handschriftlichen (1857/1858) und in der letzten gedruckten Fassung (1882). Stuttgart/Bad Cannstatt 1977; Heidegger, Martin: Sein und Zeit. 16. Aufl. Tübingen 1986; Gadamer, Hans-Georg: Hermeneutik I. Wahrheit und Methode. Grundzüge einer philosophischen Hermeneutik. 6. Aufl. Tübingen 1990.

$46 \mathrm{Vgl}$. zum Intentionalismus hier zusammenfassend Spoerhase, Carlos: Autorschaft und Interpretation. Methodische Grundlagen einer philologischen Hermeneutik. Berlin/New York 2007; Zur produktiven Inversion des Kampfbegriffes des >intentionalen Fehlschlusses< nach Beardsley, Monroe C./Wimsatt Jr., William K.: »The Intentional Fallacy«. In: The Sewanee Review 54 (1946), S. 468-488, wieder in: Wimsatt, William K.: The Verbal Icon. Studies in the Meaning of Poetry. Lexington 1954, S. 3-18, in dieser Diskussion maßgeblich Danneberg, Lutz/Müller, Hans-Harald: »Der intentionale Fehlschluß - ein Dogma? Systematischer Forschungsbericht zur Kontroverse um eine intentionalistische Konzeption in den Textwissenschaften«. In: Zeitschrift für allgemeine Wissenschaftstheorie 14 (1983), S. 103-137 (Teil I) u. S. 376-411 (Teil II). Vgl. auch Köppe, Tilmann/Kindt, Tom: »Conceptions of Authorship and Authorial Intention«. In: Gillis Dorleijn u. a. (Hg.): Authorship Revisited: Conceptions of Authorship around 1900 and 2000. Leuven u. a. 2010, S. 213-227.
}

47 Vgl. Rorty, Richard: Der Spiegel der Natur. Eine Kritik der Philosophie. Frankfurt a. M. 1981. 
tik Gadamers hier wohl kaum zum Kronzeugen für die historische Offenheit und Dynamik von postmodernen Sinnbildungsprozessen herangezogen werden können. ${ }^{47}$

Wenn man den Traditionalismusvorwurf im Sinne dieser Wende auch schon von vornherein über das Autorkonzept auflösen möchte, so könnte man bereits auf Schleiermachers klassisches Diktum verweisen, es gelte, die Rede »besser zu verstehen als ihr Urheber ${ }^{48}{ }^{48}$ enthält doch dieses Besserverstehen bereits die Paradoxie einer vertiefenden Abhebung von der postulierten Sinnstiftung. In der deutschen akademischen Rezeption musste das Innovationspotential dieser semantischen Basisparadoxie indes im kritischen Gegenzug zur Gadamerschen Hermeneutik eigens betont werden: im rezeptionsästhetischen Begriff der >Horizontabhebung< im Zusammenhang der Diskussionen im Umkreis der Forschergruppe >Poetik und Hermeneutik $<.^{49}$ Weil die Horizontabhebung als kritischer Gegenbegriff zur Horizontverschmelzung angelegt zu sein schien, wurde damit die historische Offenheit der Hermeneutik, d.h. ihre Sensibilität für semantische Innovationen im Lektüreprozess, allerdings eher heruntergespielt als hervorgehoben. Dafür bot sich die Möglichkeit, den Verstehensbegriff literaturwissenschaftlich zu spezifizieren, weil er sich über die Dialektik von Norm und Abweichung an bestehende literaturwissenschaftliche Konzepte, insbesondere das des Formalismus anschließen oder aber in Richtung einer phänomenologisch angeleiteten, semiotischen Rezeptionsästhetik weiterdenken ließ.

Folgenreich sind diese Anschlüsse indes dahingehend, dass über sie die in der Horizontabhebung betonte Distanz, sei sie dem historisch-zeitlichen Abstand oder der modernen Rezeptionsdisposition geschuldet, zugleich in ein Verhältnis von Text und Theorie überführt wurde. Zwischen Text und Theorie entsteht nun noch einmal das gleiche Distanzverhältnis, das sich die kritische Modernität im Umgang mit ihren Gegenständen zu Gute hält, allerdings mit einer Pointe, welche die philosophische Hermeneutik gerade nicht kennt. Insbesondere die philosophische Hermeneutik Gadamers versteht sich nicht als radikal distanzierte, differenzierende Theorie H. Von einer Theorie der Interpretation ist hier vielmehr nur in einem paradoxen Sinne zu reden: Denn Hermeneutik ist immer zugleich Theorie und Praxis, sie ist eine selbstreflexive Methode, die um die Unerfüllbarkeit der Theorieversprechen in der Praxis ebenso weiß, wie sie die Blindheit der Praxis ohne theoretische Reflexion anerkennt. Als Gegenstück zu diesem Methodenverständnis lassen sich die beharrlichen hermeneutischen Meditationen von Paul Ricœur ansehen, die, anstatt die Hermeneutik durch einzelne Theorien einzuschränken, genau umgekehrt verschiedene Theorieansätze aus Psychologie, Narratologie und Strukturalismus aus Sicht des hermeneutischen Methodengedankens kritisierte..$^{50}$

\footnotetext{
48 Schleiermacher (s. Anm. 3), S. 94.

49 Vgl. Jauss, Hans Robert: Ästhetische Erfahrung und literarische Hermeneutik. Bd. 1: Versuche im Feld der ästhetischen Erfahrung. München 1977; Iser, Wolfgang: Der Akt des Lesens. Theorie ästhetischer Wirkung. 4. Aufl. München 1994.

50 Vgl. die Kritiken in Ricœur, Paul: Hermeneutik und Strukturalismus. Der Konflikt der Interpretationen I. München 1973; Ders.: Hermeneutik und Psychoanalyse. Der Konflikt der Interpretationen II. München 1974; Ders.: Zeit und Erzählung. Band. I: Zeit und historische Erzählung. Aus dem Französischen von Rainer Rochlitz. Band. II: Zeit und literarische Erzählung. Aus dem Französischen von Rainer Rochlitz. Band. III: Die erzählte Zeit. Aus dem Französischen von Andreas Knop. München 1988-1991 zur Narratologie besonders Bd. II. Zum hermeneutischen Selbstverständnis besonders ders. Die lebendige Metapher.
} 
An genau dieser Stelle führt die hohe Theorieaffinität der neueren Diskussionen um Literatur, Text und Medien zu einem gespaltenen Verhältnis zur Hermeneutik. Wo Interpretation im technischen Sinne zur Analyse wird, ${ }^{51}$ wo sich Literaturwissenschaft vielleicht sogar im philosophischen Sinne als analytisch versteht, wirkt das hermeneutische Projekt als theoretisch defizitär. An die Stelle der hermeneutischen Distanzbeziehung tritt ein Differenzverhältnis, das es nunmehr erlaubt, hinter der Interpretation verschiedene, gegeneinander zu differenzierende Interpretationstheorien zu klassifizieren. In einer universalen Hermeneutik gehen diese dann aber nicht mehr auf. ${ }^{52}$ Der generalisierende methodische Anspruch der Hermeneutik wird damit aufgelöst - und letztlich die Legitimität ihrer Paradoxie traditionell differenzlogisch bestritten.

\section{Historisierung und neue Lektüre: Chancen des Ungleichzeitigen}

Wie prekär diese Denkweise zunächst in historischer Hinsicht ist, zeigt sich an der These, es gäbe ein >Diesseits der Hermeneutik<, dessen semantische Unmittelbarkeiten man sich dann vorzugsweise im Mittelalter erträumt. ${ }^{53}$ Mehr Jenseits der Hermeneutik als im Mittelalter war jedoch nie - die sogenannte mittelalterliche Hermeneutik, d. h. die Lehre vom drei-, oder bekannter: vom vierfachen Schriftsinn, hinter die diese These vom Mittelalter als einer symbolischen Präsens- und Prägnanzkultur glaubt zurückfallen zu können, ist epistemologisch so pauschal nicht hintergehbar. ${ }^{54}$ Denn obwohl man aus Sicht der späteren, nachaufklärerischen philosophischen Hermeneutik sagen muss, dass die mittelalterliche Kunstlehre zu den Zeichenebenen der Interpretation durch ihren normativen Anspruch jene Freiheit zur

Mit einem Vorwort zur deutschen Ausgabe. Aus dem Französischen übersetzt von Rainer Rochlitz. München 1986, sowie abschließend ders.: An den Grenzen der Hermeneutik. Philosophische Reflexionen über die Religion. Hg., übers. und mit einem Nachwort versehen von Veronika Hoffmann. Freiburg i. Br. 2008.

51 Vgl. Nassen, Ulrich: »Statt einer Einleitung. Notizen zur philologischen Hermeneutik«. In: Ders. (Hg.): Texthermeneutik. Aktualität, Geschichte, Kritik. Paderborn u. a. 1979, S. 9-22. Im gleichen Band macht sich Friedrich A. Kittler an eine Abschaffung der Hermeneutik durch eine medientheoretisch instruierte Diskursanalyse. Verstehen oder auch nur die Lektüre ganzer Werke gelten ihm als altmodisch.

52 Vgl. die Taxonomie der Interpretationstheorien bei Strube, Werner: Analytische Philosophie der Literaturwissenschaft. Untersuchungen zur literaturwissenschaftlichen Definition, Klassifikation, Interpretation, Bewertung. Paderborn 1993.

53 Vgl. Gumbrecht, Hans Ulrich: Diesseits der Hermeneutik. Die Produktion von Präsenz. Frankfurt a. M. 2004.

54 Vgl. Brinkmann, Hennig: Mittelalterliche Hermeneutik. Tübingen 1980. Zur Stellung der mittelalterlichen Hermeneutik in der Geschichte der Hermeneutik insgesamt die Skizzen von Rusterholz, Peter: »Hermeneutik«. In: Heinz Ludwig Arnold/Volker Sinemus (Hg.): Grundzüge der Literatur- und Sprachwissenschaft. Bd. 1: Literaturwissenschaft. München 1973, S. 89-114; Rusterholz, Peter: »Hermeneutische Modelle«. In: Heinz Ludwig Arnold/Heinrich Detering (Hg.): Grundzüge der Literaturwissenschaft. München 1996, S. 101-136 (weiterführend Rusterholz, Peter: »Zum Verhältnis von Hermeneutik und neueren antihermeneutischen Strömungen«. In: ebd., S. 157-177); sowie Gadamer, Hans-Georg: Art. >Hermeneutik<. In: Historisches Wörterbuch der Philosophie 3 (1974), Sp. 1061-1073; Ders.: »Klassische und philosophische Hermeneutik«. In: Ders.: Hermeneutik II. Wahrheit und Methode. Ergänzungen. Register. 2. Aufl. Tübingen 1993, S. 92-117. 
Selbstreflexion fehlt, aus dem sich der Methodenbegriff der Hermeneutik ableitet, ist sie doch gerade keine Proto-Hermeneutik.

Im Gegenteil: Sie erscheint als extrem voraussetzungsreicher Versuch einer theoretisch-systematischen Kanalisierung der hermeneutischen Freiheit, die angesichts der Wunder der Schöpfung ebenso systematisch überfordert ist wie später auf andere Weise die aufklärerische Vernunft. Demgegenüber wirkt das Postulat vom mittelalterlichen Diesseits der hermeneutischen Schwierigkeiten wie eine typisch moderne, differenzlogische Einfachheitsphantasie, die noch aus der philosophischen Position Heideggers eine Art von Fundamentalontologie herausholt, die man aus ihr nur schwer herauslesen kann, weil sie letztlich einfach anti-hermeneutisch wäre. ${ }^{55}$ Wenn es ein solches mittelalterliches Diesseits der Hermeneutik freilich gegeben hätte, auch die besagten differenzlogischen Ressentiments gegenüber der modernen Hermeneutik wären völlig im Recht.

Genau gegenläufig zu solchen theoretisch oder historisch distanzierenden Tendenzen entwickelt sich die Hermeneutik als anspruchsvolles Projekt eben erst dort weiter, wo Literaturwissenschaft ihr eigenes Verständnis jenseits der differenzlogischen Taxonomien weiterdenkt. Unter dem Einfluss der Dekonstruktion hat sich das >Entfernte Verstehen< als subtile Antwort auf die Aporien des binären Zeichendenkens erwiesen, die es im Interpretationsakt der Postmoderne nicht nur auszuhalten gilt, sondern die hier auch dialektisch aufzuheben sind.$^{56}$ Der neue Lektürebegriff, der im Zuge der Diskussionen um die Spielarten der Dekonstruktion über die Orientierung an den Differenz- und Schriftkonzepten von Jaques Derrida auf der einen und der rhetorischen Literaturauffassung von Paul de Man auf der anderen Seite aufgekommen ist, tritt in Konkurrenz zum Begriff der Textinterpretation, indem er diesen nicht etwa abweist, sondern entsprechend aufhebt: Die paradoxe Gegenläufigkeit jeder Lektüre, die sich zugleich mit und gegen den Sinn der Zeichen vollzieht und daher auch im Interpretationsakt schon ein dekonstruierendes Moment kennt, wird hier nur gleichsam invertiert.

Was dabei letztlich den Angelpunkt des hermeneutischen Anspruchs begründet, könnte sich schon, jenseits der literaturwissenschaftlichen Versuche, in der Diskussion zwischen Gadamer und Derrida gezeigt haben. Mit der Philosophie Manfred Franks, die Derridas Dekonstruktionskonzept produktiv als Neostrukturalismus kritisiert und sich auch deshalb das Etikett der Neo-Hermeneutik verdient hat, ${ }^{57}$ wird man hier nämlich fragen können, ob sich die neuere Hermeneutik und die Dekonstruktion nicht als zwei Seiten einer Medaille auffassen lassen. Es wären dieser

\footnotetext{
55 Vgl. Gumbrecht (s. Anm. 53), S. 12, 22-35, 85-93.

56 Vgl. Hamacher, Werner: »Hermeneutische Ellipsen. Schrift und Zirkel bei Schleiermacher«. In: Ulrich Nassen (Hg.): Texthermeneutik. Aktualität, Geschichte, Kritik. Paderborn u. a. 1979, S. 113-148; Ders.: Entferntes Verstehen. Studien zu Philosophie und Literatur von Kant bis Celan. Frankfurt a. M. 1998.

57 Vgl. die besagte Kritik bei Frank, Manfred: Was ist Neostrukturalismus. Frankfurt a. M. 1984. Ausgangspunkt dazu ist die Auseinandersetzung mit Schleiermacher: Ders.: Das individuelle Allgemeine. Textstrukturierung und -interpretation nach Schleiermacher. Frankfurt a. M. 1977. Zur weiteren Integration französischer Theorieeinflüsse ders.: Das Sagbare und das Unsagbare. Studien zur neuesten französischen Hermeneutik und Texttheorie. Frankfurt a. M. 1980; Ders.: Die Unhintergehbarkeit von Individualität. Reflexionen über Subjekt, Person und Individuum aus Anlaß ihrer >postmodernen< Toterklärung. Frankfurt a. M. 1986.
} 
Diskussion zufolge dann nicht die Fragen nach dem Autor, der Intention, der Tradition oder der ontologischen Grundlage, die dieser neuen, durch die Dekonstruktion gewendeten Neo-Hermeneutik als Basis dienen. Vielmehr offenbart sie sich gerade auch in der Literatur als ein zutiefst ethisches Projekt. ${ }^{58}$ Das Verstehen dreht sich um Axiologien. Über diese verspricht es, das Agonale im Streit der Interpretationen $\mathrm{zu}$ beenden. Eine gute Interpretation erscheint darum immer auch als wertvolles Lektüreergebnis.

Damit ist man wieder bei jener Lektüre angelangt, angesichts derer die neuen Leser*innen sich ihrer eigenen Orientierungsschwierigkeiten gewahr werden. Diese Orientierungsschwierigkeiten lassen sich nun nämlich als Chance begreifen, das Verstehen neu zu begründen. Dazu müsste allerdings das Modernitätsphantasma mit seinem kritischen Rationalitätsoptimismus gründlich überholt werden. Dass die neuen Leser*innen nunmehr einer Literatur begegnen, die ihnen nicht mehr habituell zugänglich ist, in denen sich das Verstehen also nunmehr von vornherein »nicht mehr von selbst ergibt, sondern in allen Punkten muss gesucht werden $«,{ }^{59}$ radikalisiert nicht nur das Problem moderner Alterität, es ist zugleich ein Einbruch der historischen Distanz in die Gegenwart. Die alten Lektüreanforderungen sind sofort gegenwärtig und neu, denn sie sind Ausdruck der typisch postmodernen Ungleichzeitigkeit verschiedener kultureller Praktiken.

Diese Ungleichzeitigkeit erfordert allerdings ein deutlich erhöhtes Maß an Selbstbeobachtung im Prozess der Interpretation. Je weniger man sich auf die gut eingespielten Selbstverständnisse des Lesens verlassen kann, umso genauer muss man die Wege des eigenen Verstehens in jedem Schritt bedenken. Es reicht nicht, nur den Text zu lesen, man muss sich dabei selbst dem Prozess der Lektüre unterziehen. Im alltäglichen Prozess der Interpretation ergibt sich so jene Meta-Ebene der Selbstreflexion erneut, auf die das Methodenverständnis der Hermeneutik zwischen Theorie und Praxis immer schon hinauswollte - freilich nicht als philosophische Hermeneutik post rem, sondern als literarische Hermeneutik in re. Ihre Verschiebung wäre nicht erst in den Alteritätseffekten von Texten und jenen ästhetischen Objekten angelegt, die bei ihrer Lektüre hervorgebracht werden. Sie wäre nun schon im neuen Leser* bzw. der neuen Leserin selbst situiert, im Staunen, in der Faszination und der Verwunderung über die eigenen, noch fremden interpretatorischen Möglichkeiten. Welche Wege der hermeneutischen Selbstreflexion in der Praxis dazu bislang vielleicht schon beschritten sind, möchte das Heft der Zeitschrift für Literaturwissenschaft und Linguistik erkunden.

Funding Open Access funding enabled and organized by Projekt DEAL.

Open Access Dieser Artikel wird unter der Creative Commons Namensnennung 4.0 International Lizenz veröffentlicht, welche die Nutzung, Vervielfältigung, Bearbeitung, Verbreitung und Wiedergabe in jeglichem Medium und Format erlaubt, sofern Sie den/die ursprünglichen Autor(en) und die Quelle ordnungsgemäß nennen, einen Link zur Creative Commons Lizenz beifügen und angeben, ob Änderungen vorgenommen wurden.

\footnotetext{
58 Vgl. Derrida, Jacques: »Guter Wille zur Macht [I]. Drei Fragen an Hans-Georg Gadamer«; »Guter Wille zur Macht [II]. Die Unterschriften interpretieren [Nietzsche/Heidegger]«. In: Philippe Forget (Hg.): Text und Interpretation. Deutsch-französische Debatte. München 1984, S. 56-58, S. 62-77.

59 Vgl. Schleiermacher (s. Anm. 3), S. 92, im Original hervorgehoben.
} 
Die in diesem Artikel enthaltenen Bilder und sonstiges Drittmaterial unterliegen ebenfalls der genannten Creative Commons Lizenz, sofern sich aus der Abbildungslegende nichts anderes ergibt. Sofern das betreffende Material nicht unter der genannten Creative Commons Lizenz steht und die betreffende Handlung nicht nach gesetzlichen Vorschriften erlaubt ist, ist für die oben aufgeführten Weiterverwendungen des Materials die Einwilligung des jeweiligen Rechteinhabers einzuholen.

Weitere Details zur Lizenz entnehmen Sie bitte der Lizenzinformation auf http://creativecommons.org/ licenses/by/4.0/deed.de. 